\title{
Benefits of endoscope-assisted microsurgery in the management of pediatric brain tumors
}

\author{
Mohamed A. El Beltagy, MD,1,2 and Mostafa M. E. Atteya, MD' \\ ${ }^{1}$ Neurosurgery Department, Children's Cancer Hospital Egypt (CCHE-57357), Cairo; and Neurosurgery Department, Kasr \\ Al-Ainy School of Medicine, Cairo University, Cairo, Egypt
}

\begin{abstract}
OBJECTIVE Microsurgical and endoscopic techniques are vastly utilized in brain tumor surgery. Combining both techniques in the same procedure has different forms and applications. The aim of this work was to discuss the usefulness and describe the technical benefits of endoscope-assisted microsurgery (EAMS) in treating pediatric brain tumors in various anatomical locations.
\end{abstract}

METHODS The medical records of 106 children who had undergone EAMS for brain tumors at Children's Cancer Hospital Egypt (CCHE-57357) between January 2009 and January 2017 were reviewed. The patients' ages ranged from 1 to 16 years (mean age 7.5 years). Technical variations, difficulties, complications, strategies, and extent of resection were addressed according to anatomical location.

RESULTS In general, EAMS enabled closer inspection of tumor extension and surrounding vital structures, especially in the hidden corners not appreciable by the microscope alone, such as tumors in the internal auditory canal and cerebellopontine angle contents in 14 cases, all of which were totally excised, and the undersurface of the optic apparatus in 65 craniopharyngiomas. Total excision was achievable in 51 of the 65 craniopharyngiomas; residual tumor was intentionally left behind under endoscopic guidance in the remaining 14 patients to ensure better hypothalamic function. Vision improved in 15 of 16 patients who initially presented with visual defects. Only 4 patients had new-onset postoperative endocrinopathies. For intraventricular tumors, EAMS allowed earlier recognition of tumor pedicle and, hence, earlier control of the blood supply of the tumor and safer total excision of 12 lateral ventricle, 6 pineal and third ventricle, and 9 fourth ventricle tumors. The tandem use of the endoscope and microscope enabled safer tumor dissections that were performed with more confidence in situations in which pure microscopic excision was either not achievable or less safe. Technical strategies, pitfalls, difficulties, and precautions were categorized and described per tumor location.

CONCLUSIONS EAMS of pediatric brain tumors is a promising, user-friendly tool that complements microsurgery in the management of these complex lesions. The benefits of $2 \mathrm{D}$ endoscopy are added to the benefits of stereoscopic perception. EAMS is especially helpful during the removal of different complex pediatric brain tumors. Simultaneous or tandem endoscopic and microscopic approaches may have the potential for better functional outcomes through better visualization and preservation of vital structures in corners that are hidden from the microscope.

https://thejns.org/doi/abs/10.3171/2020.10.FOCUS20620

KEYWORDS endoscope-assisted; endoscopic-assisted; keyhole; microsurgery; neuroendoscopy; skull base

$\mathrm{T}$ HE earliest neuroendoscopic procedures were performed and published by L'Espinasse, who coagulated the choroid plexus endoscopically, and by Doyen, who utilized an endoscope in posterior fossa surgery in 1917..$^{-3}$ Those were followed by further endoscopic procedures by Dandy, Fukushima, and Prott. ${ }^{4-6}$

Hopf and Perneczky introduced the concept of "endoscope-assisted microsurgery" (EAMS) in which the surgery is primarily performed under the operative microscope in addition to the endoscope, which serves as an adjunct to the microscopic manipulations, in contrast to "endoscope-controlled neurosurgery," in which the endoscope is utilized as the primary operative tool.7 In endoscope-assisted techniques, the medium for surgery is air, and the tools utilized are primarily microsurgical, whereas in purely endoscopic procedures, the medium for surgery

ABBREVIATIONS CPA = cerebellopontine angle; EAMS = endoscope-assisted microsurgery; GTR = gross-total resection; IAC = internal auditory canal; NTR = near-total resection; STR = subtotal resection.

SUBMITTED July 3, 2020. ACCEPTED October 8, 2020.

INCLUDE WHEN CITING DOI: 10.3171/2020.10.FOCUS20620. 
is water and the tools utilized are transendoscopic. ${ }^{6}$ The merits of using the endoscope in parallel with the microscope include providing a better operative field of vision, less brain retraction, and better exposure through better illumination and wider angles of vision, especially in skull base surgery. 8,9

The aim of this work was to discuss the usefulness and technical details of combined endoscopic and microsurgical approaches in treating different pediatric brain tumors in various intracranial locations. Each tumor location was individually assessed, and a conclusive analysis of the technique is provided.

\section{Methods}

We retrospectively reviewed the medical records of children operated on for brain tumors at Children's Cancer Hospital Egypt (CCHE-57357) from January 2009 to January 2017. One hundred six patients had undergone EAMS for excision of different supratentorial and infratentorial brain tumors. Table 1 shows the studied cases according to tumor pathology, tumor location, and extent of resection.

The patients' ages ranged from 1 to 16 years (mean age 7.5 years), and the male-to-female ratio was 1.7:1. Intraoperative techniques, difficulties, benefits, and variations of the techniques were reviewed, together with the extent of tumor resection and complications.

Microneurosurgical techniques were utilized for the initial approaches. The operative endoscope was introduced to enable a more detailed appreciation of the relation of the tumor to the surrounding vital structures and to avoid blind traction over these structures. A rigid endoscope with $0^{\circ}, 30^{\circ}, 45^{\circ}$, or $70^{\circ}$ angles of vision was utilized accordingly. The benefits and technical difficulties of endoscopic assistance were assessed for each tumor location. The operative setup and device handling techniques are also described.

\section{Results}

\section{Timing of Endoscopic Intervention}

Following the initial routinely performed microsurgical approach, the surgeon had to assess the operative situation and choose the best strategy for approaching the tumor. This decision varied widely and depended on the size and location of the tumor. Sometimes the tumor was large enough to hinder the initial introduction of the endoscope, and the surgeon had to perform internal debulking first to create some room for the endoscope. The endoscope was introduced later to assess the boundaries and relationships of the tumor, a technique that was feasible in tight or crowded locations such as the cerebellopontine angle (CPA) and suprasellar region. In other situations, the endoscope had to be introduced first to cauterize a vascular tumor pedicle and to facilitate further microscopic excision, which was feasible in some choroid plexus tumors, for instance. In some instances, combined simultaneous or alternative tandem endoscopic and microscopic assessment and manipulation were performed in areas extending to hidden corners of the operative field.
TABLE 1. The study cases according to tumor pathology, tumor location, and extent of resection

\begin{tabular}{lrrrr}
\hline & No. of Cases & GTR & NTR & STR \\
\hline Craniopharyngioma & 65 & 51 & 11 & 3 \\
\hline CPA tumor & 14 & 14 & & \\
\hline Epidermoid & 5 & 5 & & \\
\hline Ependymoma & 5 & 5 & & \\
\hline Vestibular schwannoma & 4 & 4 & & \\
\hline Pineal/posterior 3rd ventricle & 6 & 5 & 1 & \\
\hline Pineocytoma & 2 & 2 & & \\
\hline Pineoblastoma & 3 & 2 & 1 & \\
\hline Neurocytoma & 1 & 1 & & \\
\hline Lateral ventricle & 12 & 11 & 1 & \\
\hline Choroid plexus carcinoma & 4 & 3 & 1 & \\
\hline Choroid plexus papilloma & 5 & 5 & & \\
\hline Ependymoma & 3 & 3 & & \\
\hline 4th ventricle & 9 & 8 & 1 & \\
\hline Ependymoma & 5 & 4 & 1 & \\
\hline Astrocytoma & 2 & 2 & & \\
\hline Medulloblastoma & 2 & 2 & & \\
\hline Total & 106 & 89 & 14 & 3 \\
\hline
\end{tabular}

\section{Handling the Endoscope for EAMS}

There are three options for holding the endoscope in endoscope-assisted techniques, detailed as follows. 1) The surgeon holds the endoscope in one hand. If enough room is created within the surgical cavity, the surgeon does not need to hold any instruments in their other hand and can carefully inspect the tumor, explore the boundaries and walls, and discover hidden corners and remnants. If there is not enough room, the surgeon enhances the inspection with the aid of an instrument in the other hand. 2) The surgeon lets the assistant hold the endoscope in a dynamic fashion following the surgeon's lead in different areas of the surgical bed. This gives the surgeon the opportunity to manipulate the tumor with one instrument in each hand. This technique, however, requires a great deal of caution, communication, and mutual understanding between the surgeon and the assistant. 3) The endoscope is fixed to the operating table by a rigid multijointed arm. However, greater precautions should be taken to avoid any unnecessary or jerky movements within the rigid arm perimeter, and the operating table in general. Figure 1 illustrates the different methods of handling the endoscope in endoscope-assisted techniques.

In some circumstances, the surgical microscope is adjusted slightly away from the operative field to provide space that allows the surgeon to hold and manipulate the endoscope and instruments while compensating for the increased working distance with a proper zoom-in of the microscopic optics.

\section{Endoscopic Inspection and Assessment Versus Endoscopic Surgical Manipulation}

Each time endoscopic assistance is used, the surgeon 

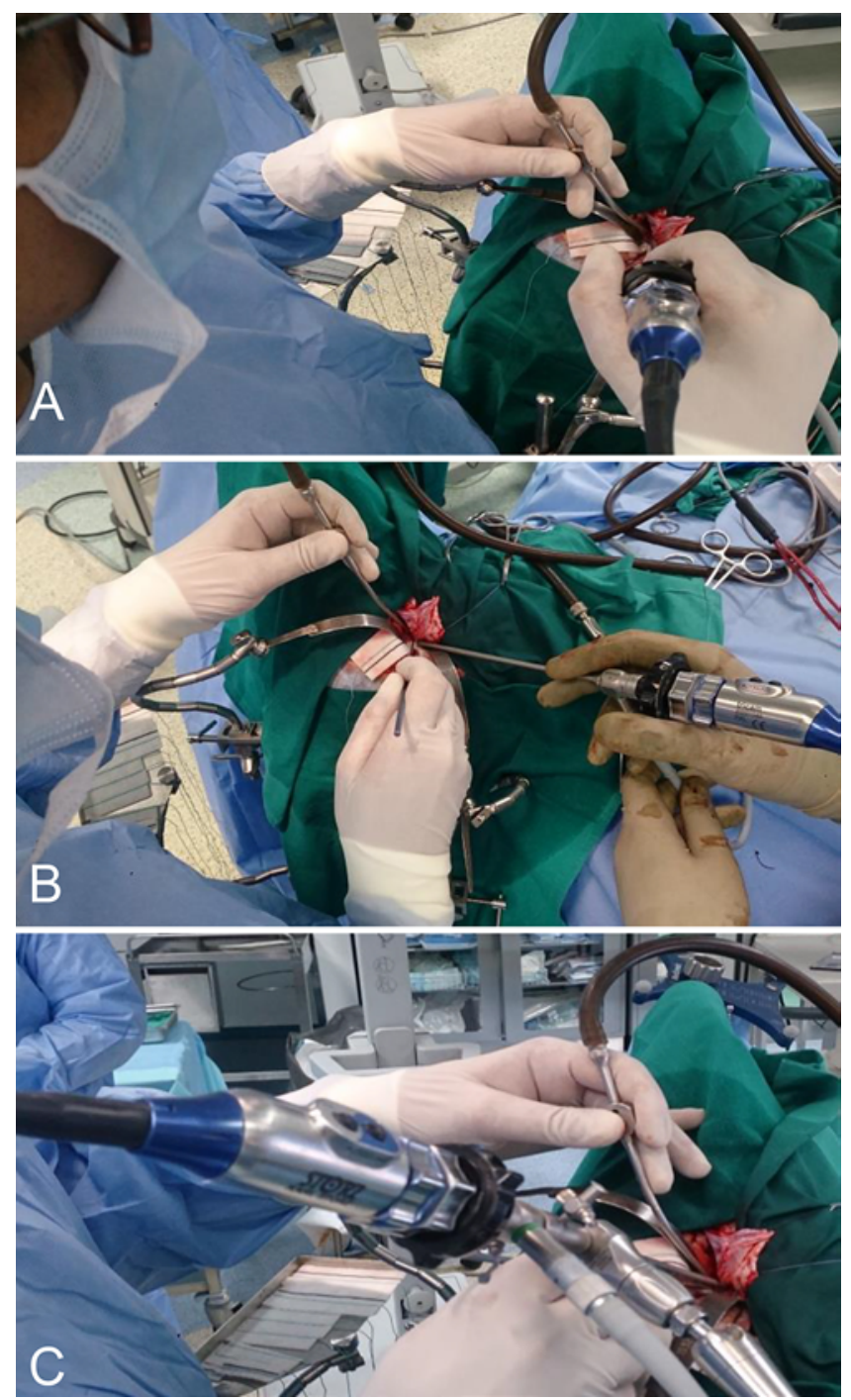

FIG. 1. Technical handling of the endoscope in endoscope-assisted techniques. A: The surgeon holds the endoscope in one hand and another instrument, such as microsuction or microdissector, in the other. $B:$ The endoscope is held dynamically by the assistant while the surgeon uses both hands in harmony with the assistant. C: The endoscope is fixed on a rigid multijointed endoscope fixator in a proper part of the operative field, and the surgeon is able to use both hands.

has to determine whether the aim is to inspect and check the surgical field only or if the tumor needs to be manipulated and the hidden and inaccessible remnants resected. This way of thinking helps the surgeon choose the best strategy for making good use of both hands and the assistant's hands as well. Is there enough room to inspect with uninterrupted lines of vision, or is additional instrumented manipulation such as by a microdissector, a micropunch, or suctioning required?

For inspection and assessment purposes, the surgeon can hold the endoscope in one hand and a suction tip in the other hand. Alternatively, when manipulation is required, the assistant can hold the endoscope, thus giving the surgeon the freedom to dissect and expose planes with multiple microsurgical instruments. Also, the surgeon can hold the endoscope with one hand and an instrument such as a micropunch in the other hand; this, of course, requires great hand stability and maximum caution. Another option is to fix the endoscope in a rigid multijointed endoscope holder and operate freely. No endoscope-related morbidities or mortalities were encountered in the studied cases.

\section{Role of the Assistant}

Only a well-trusted assistant with a steady hand can be of help in endoscope-assisted techniques. In addition to the roles mentioned above, the assistant has a critical role to play, which is close monitoring of the vital neurovascular structures during endoscopic application and manipulation. This is because the surgeon is focusing only on the region of interest, and another keen eye is required to monitor other structures along the entire trajectory of the endoscope's shaft. This is especially crucial in the CPA and in suprasellar approaches. Thus, both the surgeon and assistant need to work in harmony to ensure a maximally safe operative environment for the endoscope-assisted approach and to avoid neurovascular injuries. The trajectory of the endoscope shaft and its tip should be continuously monitored by the assistant at all times through the microscope's binoculars, and also by intermittent direct naked eye inspection of the entire surgical corridor from outside the microscope binoculars while the surgeon is performing endoscopic maneuvers.

\section{General Benefits of EAMS in the Studied Cases}

In all cases, endoscopic assistance enabled closer inspection of the extensions and relations of the tumor to the surrounding structures, especially in the hidden corners not appreciable by microscopic vision, such as the internal auditory canal (IAC) and the undersurface of the optic apparatus. If the tumor is small and there are potential tissue planes, the endoscope is introduced early in the procedure to verify anatomy, planes, and hidden corners. However, if the tumor is large or there is no space to introduce the endoscope, a microscope is used first to create planes during ongoing dissection, followed by inspection and assessment by the endoscope. Tandem utilization of microscope and endoscope-assisted dissection and tumor excision enabled a safer and more confident excision of tumors in critical areas and hidden corners of the operative field.

\section{EAMS for Craniopharyngioma}

Among 65 children with craniopharyngiomas treated using EAMS techniques, gross-total resection (GTR) was achieved in $51(78.5 \%)$. Near-total resection (NTR) with residual tumors less than $1 \mathrm{~cm}$ and subtotal resection (STR) with residual tumors more than $1 \mathrm{~cm}$ were performed in 11 and 3 patients, respectively. Sixteen patients initially presented with visual deterioration; 15 of these 16 patients showed visual improvement. No patient had postoperative visual deterioration.

Endoscopic assistance enabled a more detailed appreciation of the tumor's relationship to the undersurface of the optic apparatus, hypothalamus, pituitary stalk, perforators, tumor-pituitary stalk interface, and Liliequist membrane (Figs. 2 and 3). Residual tumor was intentionally left 

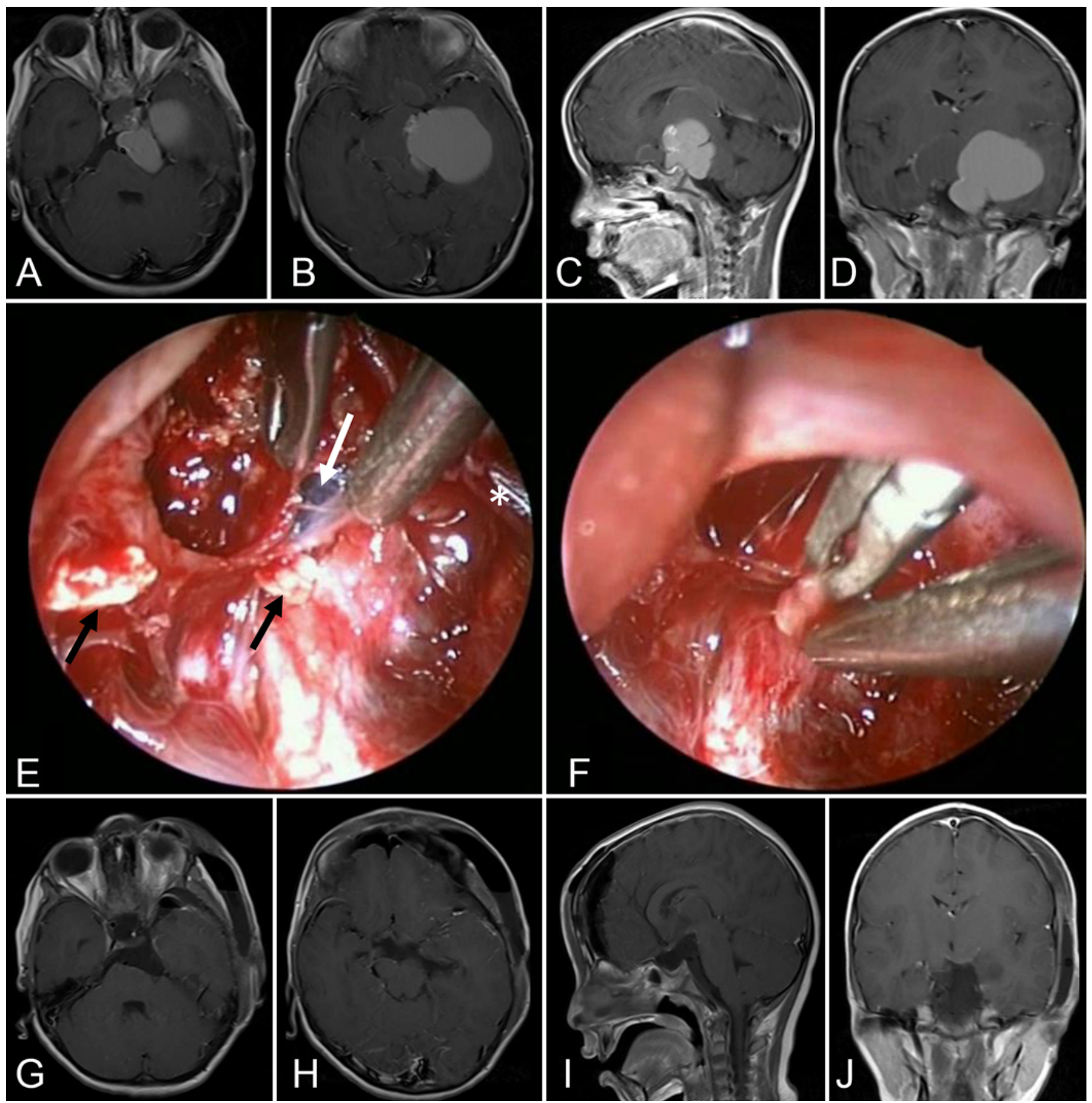

FIG. 2. EAMS during resection of a craniopharyngioma. A-D: Preoperative axial (A and B), sagittal (C), and coronal (D) contrastenhanced MR images showing a large craniopharyngioma with complex extensions into the hypothalamus interpeduncular, crural, and prepontine cisterns and middle cranial skull fossa. E: Intraoperative deep endoscopic view showing small tumor residuals (black arrows) attached to the Liliequist membrane (white arrow). Note that the pituitary stalk (asterisk) is preserved during EAMS. In this case, the surgeon is using both hands to manipulate the residual tumor with a pituitary microrongeur and a suction tip, and the endoscope in this case is held in a dynamic fashion in the depth of the surgical field by the assisting surgeon. F: A piece of the tumor is being removed by the pituitary microrongeur, after it was determined to be dissectible as confirmed by the endoscopic manipulation. G-J: Early postoperative axial ( $\mathrm{G}$ and $\mathrm{H})$, sagittal $(\mathrm{I})$, and coronal $(\mathrm{J})$ contrast-enhanced MR images showing total tumor excision.

behind, as mentioned above, to allow the child to live with better hypothalamic function.

Residual tumor in the hidden corners that could not be assessed by the surgical microscope was easily assessed by the endoscope; a $30^{\circ}$ endoscopic lens was especially helpful in this regard.

Even when GTR was not possible, endoscopic assistance enabled leaving the smallest residual, thus decreas- 

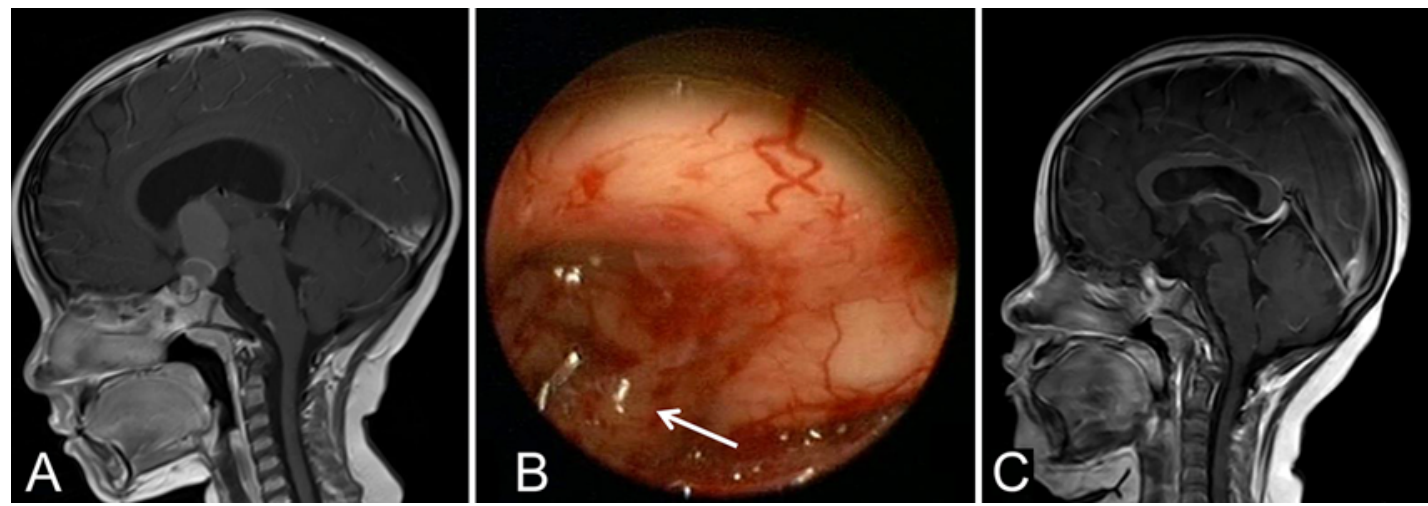

FIG. 3. Residual craniopharyngioma detected on the undersurface of the optic chiasm after assumed total excision by the microscope. A: Preoperative sagittal contrast-enhanced MR image showing a large craniopharyngioma. B: Residual tumor (arrow). C: Postoperative sagittal contrast-enhanced MR image showing total tumor excision.

ing the adjuvant disease burden and preserving functionality. In craniopharyngiomas with intraventricular extension, endoscope-assisted excision of intraventricular tumor portions after fenestration of the lamina terminalis obviated the need for a double approach or transcallosal approach, in addition to avoidance of blind traction over the hypothalamus, pituitary stalk, and ventral surface of the optic apparatus, together with a better chance of preservation of the perforating vessels.

One patient experienced a transient oculomotor nerve palsy that gradually improved over a 3-month duration. Endocrine deficits were diagnosed preoperatively in 15 patients. Postoperatively, 19 patients presented with different varieties of endocrine disturbance. Postoperative subdural collections occurred in 7 patients and were managed conservatively, except in 1 patient, who was managed by subdural low-pressure shunting.

\section{EAMS for CPA Tumors}

Fourteen children in this study had CPA tumors. There were 5 epidermoids, 5 ependymomas, and 4 vestibular schwannomas. GTR was achieved in all of these tumors. Intraoperative neurophysiological monitoring techniques were utilized in 7 cases.

EAMS improved tumor exposure, especially in hidden corners such as the lateral parts of the IAC, thus enabling safe GTR in all cases (Fig. 4 and Table 1). Early detection of the facial nerve is an added advantage of EAMS, together with better assessment of the IAC, enabling panoramic, flexible, safe, and well-visualized working channels between the lower cranial nerves while preserving their function. In addition, EAMS minimized the amount of cerebellar retraction needed for sufficient exposure and enabled a reduced craniotomy size and better identification of opened mastoid air cells to avoid CSF leaks. One patient had transient mild postoperative facial nerve palsy that improved 3 weeks postoperatively. Neuronavigation and intraoperative neuromonitoring were helpful adjuncts in these tumors.

\section{EAMS for Pineal and Posterior Third Ventricle Tumors}

Six children with pineal/posterior third ventricle tu- mors were operated on with EAMS techniques. Three patients had pineoblastomas (GTR was achieved in 2 of them and NTR in the remaining patient). Two patients had pineocytomas and 1 had a neurocytoma; GTR was successfully performed in all.

Early pure endoscopic inspection and assessment of the lesions, vascular supply, and relationship to nearby structures were performed. Early control of tumor vascularity made further tumor manipulations and excisions much easier and safer (Fig. 5). In some cases, an endoscopic transcortical trajectory-guided microsurgical approach was utilized to enable the earliest possible access to the tumor pedicle and vascular control.

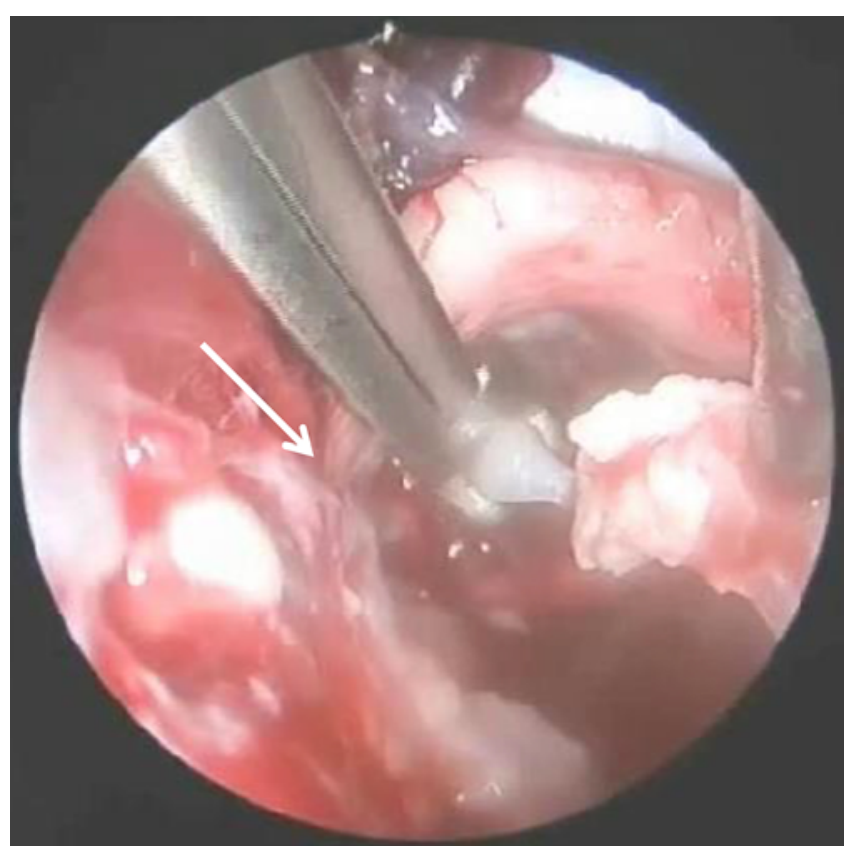

FIG. 4. A case of a CPA epidermoid in which tumor residual is removed from the IAC (arrow) with endoscopic assistance. The surgeon uses both hands to manipulate the tumor residual, and the assistant holds the endoscope in a dynamic fashion in harmony with the surgeon's movements. 

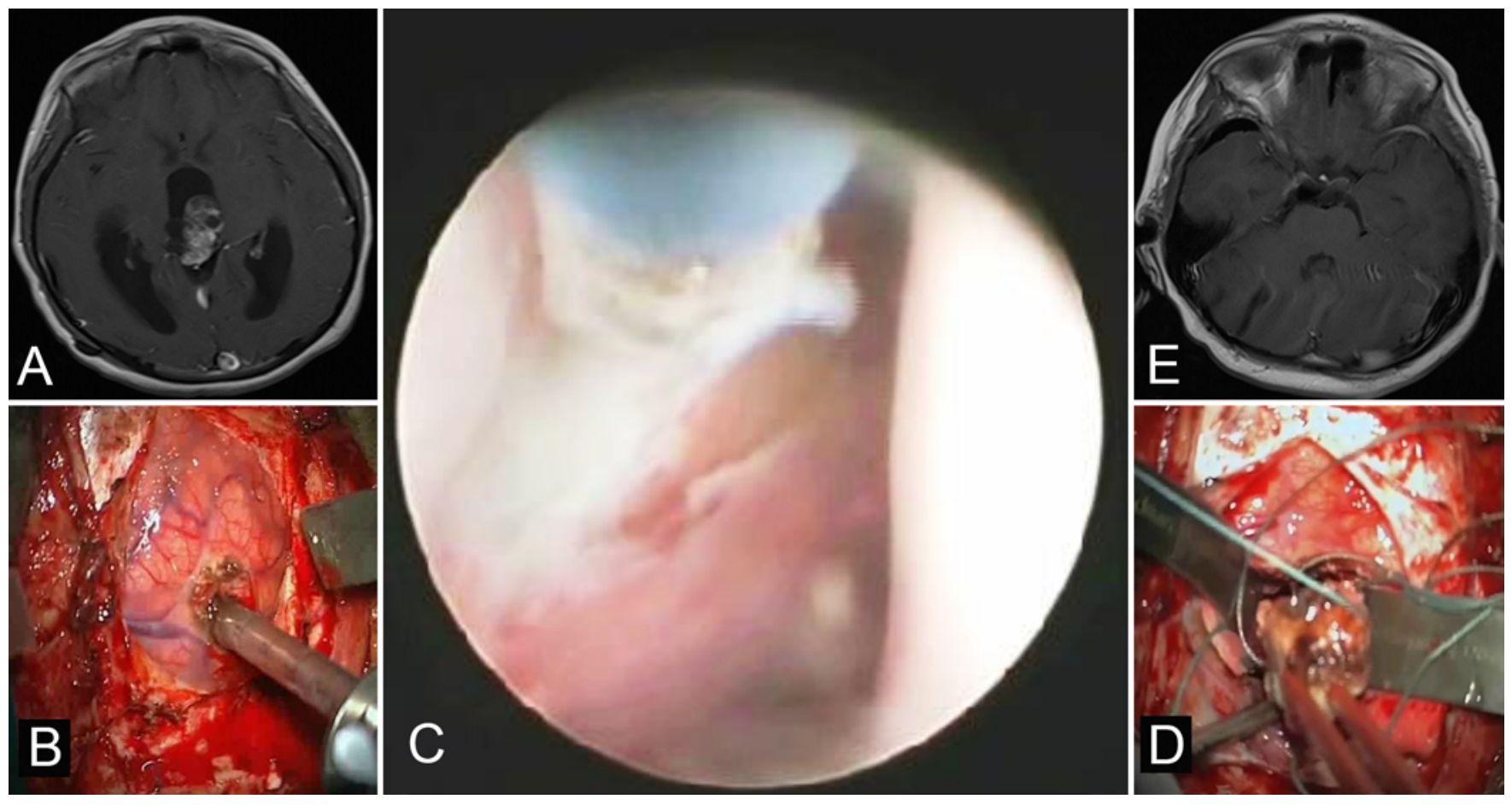

FIG. 5. A case of a posterior third ventricle neurocytoma. A: Preoperative axial contrast-enhanced T1-weighted MR image. B: Intraoperative photograph showing early transcortical introduction of the endoscope. C: Early endoscopic coagulation of the tumoral blood supply. D: Total microscopic excision of the tumor in one piece was made easier after endoscope-assisted blood supply control, rendering the tumor softer and less vascular. E: Postoperative axial contrast-enhanced T1-weighted MR image showing total tumor excision.

\section{EAMS for Surgery of Lateral Ventricle Tumors}

Twelve children with lateral ventricle tumors underwent EAMS. Four patients had choroid plexus carcinoma, 5 had choroid plexus papilloma, and 3 had ependymoma. GTR was achieved in all patients except 1 patient with a choroid plexus carcinoma, for which NTR was achieved. EAMS allowed early recognition of the tumor's pedicle (Fig. 6), early control of the tumoral blood supply, and different angles of vision and flexible trajectory changes, which enhanced closer inspection of the boundaries of large tumors and the creation of tumor cleavage planes within the narrow ventricular spaces. Neuronavigation and intraoperative ultrasound were helpful operative adjuncts for these tumors.

\section{EAMS for Surgery of Fourth Ventricle Tumors}

In 9 patients with fourth ventricle tumors, EAMS was utilized to assess the relation of the tumors to the brainstem or the lateral recesses and to control tumor resection safely. Of these patients, 5 had ependymomas, 2 had astrocytomas, and 2 had medulloblastomas. GTR was achieved in all cases except 1 case of an ependymoma that was attached to the lateral aspect of the brainstem, for which NTR was performed.

EAMS enabled safe assessment of the tumor's relationship to structures throughout the entire fourth ventricle and its lateral recesses and enabled a minimal amount of cerebellar retraction for tumor manipulation, thus achieving GTR under panoramic view and excellent illumination especially in cases in which the lesion invaded the lateral brainstem and lateral recesses.

\section{Discussion}

\section{Microscope Versus Endoscope}

Hidden tumor portions behind the naturally existing barriers of the skull base and neurovascular structures are sometimes difficult to assess using a surgical microscope, due to the straight visual lines of the microscope. Thus, the addition of an endoscope theoretically aids in checking the different angles and corners of the operative field, with improved visualization. ${ }^{10}$ However, the endoscope provides 2D images that can attain a sense of depth perception or a pseudo-third dimension by the effect of dynamic magnification and surgical exploration. Also, the endoscope takes up a significant amount of space in the operative field and requires extra attention to neurovascular elements along the surgical track. ${ }^{11}$

\section{Advantages}

Endoscopic assistance in microsurgical procedures enables minimization of brain retraction and reduction of the amount of work that has to be done to the bone, including the bone flap size and the extent of skull base drilling. It also provides better illumination, clearer appreciation of the corners of the operative field, and closer inspection of the neurovascular elements that are not easily visualized. Visualization of these elements is of particular importance 

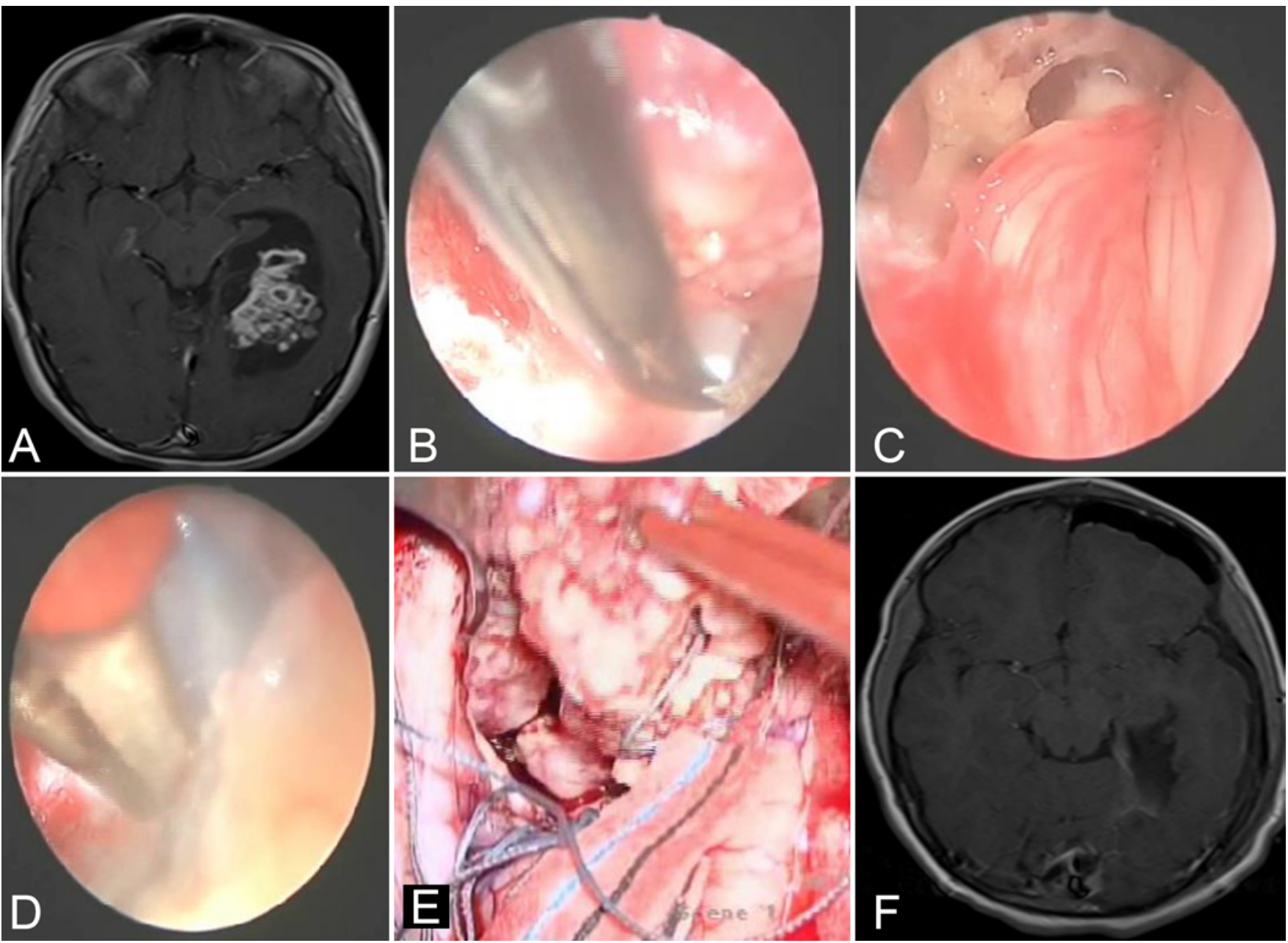

FIG. 6. A case of intraventricular meningioma. A: Preoperative axial contrast-enhanced T1-weighted MR image showing a left occipital horn tumor. B: An early endoscope-assisted cleavage plane is created with microscissors. C: Early endoscope-assisted vascular tumor pedicle exposure. D: Bipolar coagulation of the vascular tumoral supply under endoscopic guidance. E: Microscopic excision of the devascularized tumor. F: Early postoperative axial contrast-enhanced T1-weighted MR image showing total tumor excision.

in skull base tumors, where the straight optical lines of the operating microscope are usually challenged by neurovascular structures en route, large tumors, and conservative craniotomies. ${ }^{10}$

In the current study, the endoscope-assisted techniques proved helpful in assisting safer tumor excision in different regions, especially those in the suprasellar and intraventricular locations. It is impressive and interesting that in many circumstances a hidden tumor residual was detected by endoscopic inspection despite total tumor excision being assumed by microscopic techniques. Also, early blood supply control is a big advantage of endoscopic assistance, especially in choroid plexus tumors.

\section{Complications and Limitations}

The major drawbacks of the endoscope include the lack of stereopsis, which means that a true 3D vision cannot be attained, and the motion parallax, which gives the impression that closer objects move faster than remote ones. The fish-eye image distortion contributes to some amount of false 3D appreciation. ${ }^{10,11}$

Neurovascular injuries have been reported during endoscope-assisted procedures. For example, the oculomotor nerve within the free edge of the tentorium can be touched by the shaft of the endoscope, especially when placed through the carotid-oculomotor triangle. The facial nerve should also be well monitored in CPA approaches to avoid injuries that may even require nerve suturing..$^{12}$ No endoscope-related deaths were encountered in the studied cases. One patient had postoperative transient oculomotor palsy. The endoscope was introduced through the carotidoculomotor triangle in this case; however, no intraoperative events were recorded in this regard. To minimize the risk of endoscope-associated injuries, the neurovascular structures in the operative field should always be monitored through the operating microscope, especially when using the $70^{\circ}$ viewing endoscopic angle in the CPA. Regular irrigation of the operative field is usually enough to 
prevent the minimal risk of thermal neurovascular injuries. $^{12}$

\section{Operative Stages of Endoscopic Assistance}

We utilized endoscopic assistance early in some cases or during stages of the tumor excision and in the final inspection for tumor residual. In select cases, the surgical corridor and working space can be widened for a given approach by the "on-demand" endoscopic assistance. The EAMS is usually required in select parts of the middle of the surgery. ${ }^{13}$

\section{Technical Specifications}

There are several technical variations of the optimal operative setup, which gives flexibility in image quality and the pattern of image display. Various systems exist, and they differ in the display patterns. These include image injection and the picture-in-picture technique where both the endoscope and microscope feeds are displayed on the same screen, whether stand-alone or mounted to the head. ${ }^{14-16}$

In the current study, we managed to position the standalone monitors in well-adjusted locations that were easy for the surgeons to observe without exerting any effort to move their head or neck or having them move outside the perimeter of the operating microscope. Combining the endoscope with the microscope in the treatment of pediatric tumors reasonably combines the advantages of both and diminishes their inherent drawbacks.

This study is one of the largest series to address the use of endoscopic assistance in complex brain tumors via all major surgical approaches, through the experience of a single pediatric neurooncology center. Further comparative studies may be needed in the future to address the long-term outcomes.

\section{Conclusions}

EAMS of pediatric brain tumors is a promising userfriendly tool that complements microsurgery in the management of these complex lesions. The benefits of 2D endoscopy are added to the benefits of stereoscopic perception. EAMS is especially helpful during the removal of different complex pediatric brain tumors. Simultaneous or tandem endoscopic and microscopic approaches may have the potential for better functional outcomes through better visualization and preservation of vital structures in corners that are hidden from the microscope.

\section{References}

1. Wilkins RH, Rengachary SS, eds. Neurosurgery. McGraw Hill; 1996:539-546.

2. Gieger M, Cohen AR. The history of neuroendoscopy. In: Cohen AR, Haines SJ, eds. Minimally Invasive Techniques in Neurosurgery. Williams \& Wilkins; 1995:1-5.

3. Doyen E. Surgical Therapeutics and Operative Techniques. Vol 1. Bailliere/Tindall \& Cox; 1917:599-602.

4. Fukushima T. Endoscopy of Meckel's cave, cisterna magna, and cerebellopontine angle. Technical note. J Neurosurg. 1978;48(2):302-306.
5. Prott W. Cisternoscopy of the cerebellopontine angle. Article in German. HNO. 1974;22(11):337-341.

6. Dandy W. An operative procedure for hydrocephalus. Johns Hopkins Hosp Bull. 1922;33:189-190.

7. Hopf NJ, Perneczky A. Endoscopic neurosurgery and endoscope-assisted microneurosurgery for the treatment of intracranial cysts. Neurosurgery. 1998;43(6):1330-1337.

8. Perneczky A, Fries G. Endoscope-assisted brain surgery: part 1 -evolution, basic concept, and current technique. Neurosurgery. 1998;42(2):219-225.

9. Batay F, Vural E, Karasu A, Al-Mefty O. Comparison of the exposure obtained by endoscope and microscope in the extended trans-sphenoidal approach. Skull Base. 2002;12(3): 119-124.

10. Schroeder HW, Hickmann AK, Baldauf J. Endoscope-assisted microsurgical resection of skull base meningiomas. Neurosurg Rev. 2011;34(4):441-455.

11. Matsumoto Y, Kurozumi K, Shimazu Y, et al. Endoscopeassisted resection of cavernous angioma at the foramen of Monro: a case report. SpringerPlus. 2016;5(1):1820.

12. Hori T, Okada Y, Maruyama T, et al. Endoscope-controlled removal of intrameatal vestibular schwannomas. Minim Invasive Neurosurg. 2006;49(1):25-29.

13. Ammirati M, Kshettry VR. Endoscope-assisted microsurgery. In: Ramina R, Pires de Aguiar PH, Tatgiba M, eds. Samii's Essentials in Neurosurgery. 2nd ed. Springer; 2014.

14. Taneda M, Kato A, Yoshimine T, Hayakawa T. Endoscopicimage display system mounted on the surgical microscope. Minim Invasive Neurosurg. 1995;38(2):85-86.

15. Salma A, Ammirati M. Real time parallel intraoperative integration of endoscopic, microscopic, and navigation images: a proof of concept based on laboratory dissections. J Neurol Surg B Skull Base. 2012;73(1):36-41.

16. Kassam A, Horowitz M, Welch W, et al. The role of endoscopic assisted microneurosurgery (image fusion technology) in the performance of neurosurgical procedures. Minim Invasive Neurosurg. 2005;48(4):191-196.

\section{Disclosures}

The authors report no conflict of interest concerning the materials or methods used in this study or the findings specified in this paper.

\section{Author Contributions}

Conception and design: both authors. Acquisition of data: both authors. Analysis and interpretation of data: both authors. Drafting the article: both authors. Critically revising the article: both authors. Reviewed submitted version of manuscript: both authors. Approved the final version of the manuscript on behalf of both authors: El Beltagy. Statistical analysis: both authors. Administrative/technical/material support: both authors. Study supervision: El Beltagy.

\section{Correspondence}

Mohamed A. El Beltagy: Children's Cancer Hospital Egypt, Cairo, Egypt. beltagy_mohamed@hotmail.com. 